\title{
Specularity Removal from Imaging Spectroscopy Data via Entropy Minimisation
}

\author{
Lin $\mathrm{Gu}^{1} \quad$ Antonio Robles-Kelly ${ }^{1,2}$ \\ ${ }^{1}$ College of Eng. and Computer Science, Australian National University, Canberra ACT 0200, Australia \\ ${ }^{2}$ NICTA , Locked Bag 8001, Canberra ACT 2601, Australia
}

\begin{abstract}
In this paper, we present a method to remove specularities from imaging spectroscopy data. We do this by making use of the dichromatic model so as to cast the problem in a linear regression setting. We do this so as to employ the average radiance for each pixel as a means to map the spectra onto a two-dimensional space. This permits the use of an entropy minimisation approach so as to recover the slope of a line described by a linear regressor. We show how this slope can be used to recover the specular coefficient in the dichromatic model and provide experiments on real-world imaging spectroscopy data. We also provide comparison with an alternative and effect a quantitative analysis that shows our method is robust to changes the degree of specularity of the image or the location of the light source in the scene.
\end{abstract}

\section{INTRODUCTION}

Imaging spectroscopy can deliver an information-rich representation which can capture the properties of the materials in the scene. It is important to note that for imaging spectroscopy, the geometry of the object under study plays an important role in the image formation process. This has attracted the attention of the computer vision community for purposes of [1], edge detection and colour analysis [2] and material identification [3].

Despite effective, these methods often lack the ability to withstand the illumination variations, specularities and shadowing. This is required to process real-world imagery in an unsupervised or automatic way. For instance, the analysis in [2] was derived from the Lambertian reflection model and, hence, is not applicable to specular reflections. Fu and Robles-Kelly [4] have proposed the use of band ratios as an alternative to raw spectral bands as features for classification as a means to shading invariance. In [5], a subspace projection method for specularity-free spectral representation is presented.

For both, imaging spectroscopy and trichromatic imagery, there have been several attempts to remove specularities from images of non-Lambertian objects. Most of the efforts in the literature were devoted to modeling the effects encountered on shiny or rough surfaces. For shiny surfaces, specular spikes and lobes have to be modelled. There have been several attempts to remove specularities from images of non-Lambertian objects. For instance Brelstaff and Blake [6] used a simple thresholding strategy to identify specularities on moving curved objects. Other lines of research remove specularities by either using additional hardware [7], imposing constrains on the input images [8], requiring color segmentation [9] as postprocessing steps, or using reflectance models to account for the distribution of image brightness [10]. The main limitation of these methods is that they either rely on pre-determined setups for the image acquisition or the use of the closed form of the BRDF to characterise the specular spike and lobe.

In a related development, Novak and Shafer [11] used the color histogram to relate the shape of the objects in the scene to their roughness so as to estimate the illuminant colour. Sato and Ikeuchi [12] used the dichromatic reflection model of Shafer [13] to separate the specular reflection component from a series of color images. Umeyama and Godin [14] separated the diffuse from the specular component by maximising the probabilistic independence between these two components via Independent Component Analysis (ICA). Tan and Ikeuchi [15] have used chromaticity to separating the reflection components of textured Surfaces using a single image.

Here, we focus on the use of the dichromatic model so as to separate the specular component from the imagery. We do this based upon an entropy minisation scheme somewhat related to the work of Finlayson et al. [16], where intrinsic images are recovered by shadow removal. Note that, nonetheless both, the work presented here and that in [16] are based upon entropy measurements, they differ substantially. In [16] the entropy is minimised under the assumptions of Lambertian reflectance, approximately Planckian lighting, and fairly narrow trichromatic camera sensors. This contrasts with our method, where 
we aim at tackling the problem in imaging spectroscopy by departing from a non-Lambertian surface assumption. We do this by employing the dichromatic model presented by [13]. Moreover, we do not assume a Plankian lighting, rather apply the method in [17] so as to recover the power spectrum of the illuminant and normalise the image radiance accordingly. Once the normalised radiance is at hand, we perform a linear regression which allows the entropy minisation to be applied.

The paper is organised as follows. In the following section we introduce the dichromatic model and ellaborate on how the reflection components may be separated using a linear regression approach. In Section III we ellaborate on how the entropy can be used to recover the slope corresponding to the line described by the dichromatic model. With this slope at hand, we show how the specularities can be recovered in a straightforward manner. At the end of Section III we also ellaborate on the implementation issues arising from our approach. Experiments are presented in Section IV and conclusions provided in Section V.

\section{Separating the Reflection Components}

As mentioned earlier, here we account for specularities in hyperspectral and multispectral imaging by employing the Dichromatic model [13]. This model has long been used in physics-based vision for characterising specular reflections on non-Lambertian surfaces. The model states that the radiance $I\left(\lambda_{i}, u\right)$ at the $i^{t h}$ band centered at the wavelength $\lambda_{i}$ for a pixel $u$ in the image can be expressed as follows

$$
I\left(\lambda_{i}, u\right)=g(u) L\left(\lambda_{i}\right) S\left(\lambda_{i}, u\right)+k(u) L\left(\lambda_{i}\right)
$$

where the first term on the right-hand side corresponds for the diffuse reflection and the second term accounts for the specular reflection. Here, $L(\cdot)$ is the power spectrum of the illuminant, $S\left(\lambda_{i}, u\right)$ is the reflectance of the object surface, $g(u)$ is the shading factor and $k(u)$ is the specular coefficient.

We can give an intuitive interpretation to the model as follows. At specular pixels, i.e. where the shading factor $g(u)$ is negligible, the surface acts as a mirror, where the radiance becomes proportional to the illuminant power spectrum $L\left(\lambda_{i}\right)$. In the other hand, if the radiance is diffuse, i.e. $k(u)$ is close to zero, then the surface appears shaded and the reflectance becomes a multiplicative term on the illuminant to determine the radiance. We can easily verify by writing

$$
R\left(\lambda_{i}, u\right)=\frac{I\left(\lambda_{i}, u\right)}{L\left(\lambda_{i}\right)}=g(u) S\left(\lambda_{i}, u\right)+k(u)
$$

Once an image is acquired, the illuminant power spectrum $L(\cdot)$ can be computed using the algorithm in [17]. Moreover, we can subtract Note that, in Equation 2 , the specular component $k(u)$ is constant over all the $N$ wavelength-indexed bands. In fact, only $S\left(\lambda_{i}, u\right)$ is a function of the wavelength.

This is an important observation since it provides a way to extract the reflectance $S(\lambda, u)$. It would be noticed that, by subtracting the mean $\bar{R}(u)$ of $R(\cdot, u)$ across the wavelengths $\lambda_{i}$, from Equation 2 the specular coefficient $k(u)$ can be eliminated. Moreover, for an image region of homogeneous reflectance, $R(\lambda, u)$ the variation of radiance across pixels is governed by the shading factor $g(u)$. Since $g(u)$ is independent of the wavelength, it can be removed via normalisation.

To commence, note that we can write

$$
R\left(\lambda_{i}, u\right)-\bar{R}(u)=g(u)\left(S\left(\lambda_{i}, u\right)-\frac{1}{N} \sum_{\lambda_{i}} S\left(\lambda_{i}, u\right)\right)
$$

Moreover, as mentioned above, we can use the standard deviation

$$
\tau_{R}^{2}=\sum_{\lambda_{i}}\left(R\left(\lambda_{i}, u\right)-\bar{R}(u)\right)^{2}
$$

so as to obtain the relation

$$
\frac{1}{\tau_{S}}\left(S\left(\lambda_{i}, u\right)-\bar{S}(u)\right)=\frac{1}{\tau_{R}}\left(R\left(\lambda_{i}, u\right)-\bar{R}(u)\right)
$$

Where $\tau_{S}^{2}=\sum_{\lambda_{i}}\left(S\left(\lambda_{i}, u\right)-\bar{S}(u)\right)^{2}$

The relation above further suggests that, the reflectance of the object in the scene can be computed from the image radiance $I\left(\lambda_{i}, u\right)$ once the illuminant power spectrum is available. As a direct consequence of Equations 2 and 5, we have that

$$
\begin{aligned}
R\left(\lambda_{i}, u\right) & =g(u)\left(S\left(\lambda_{i}, u\right)-\bar{S}(u)\right)+g(u) \bar{S}(u)+k(u) \\
& =g(u) \tau_{S} \hat{S}\left(\lambda_{i}, u\right)+g(u) \bar{S}(u)+k(u) \\
& =a(u) \hat{S}\left(\lambda_{i}, u\right)+b(u)
\end{aligned}
$$

where, in the last line of the above equations we have used the short hands

$$
\begin{aligned}
& b(u)=g(u) \bar{S}(u)+k(u) \\
& a(u)=g(u) \tau_{S}
\end{aligned}
$$

and introduced the variable $\hat{S}\left(\lambda_{i}, u\right)=S\left(\lambda_{i}, u\right)-\bar{S}(u)$ for the sake of brevity.

The use of these short hands permits the use of linear regression to recover the variables $a(u)$ and $b(u)$ in 

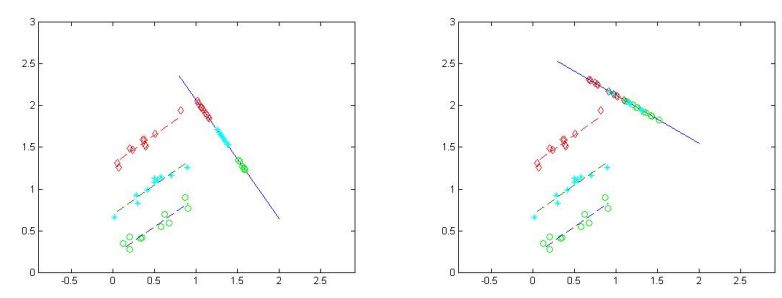

Fig. 1. Illustration of our entropy minimisation scheme. The lefthand panel shows points projected onto the line perpendicular to that with slope $c$; The right-hand panel shows the same set of points projected onto a line which is suboptimal with respect to the Shannon entropy.

Equation 6. To do this, note that, from Equation 7, we can express $b(u)$ using a line equation of the form

$$
b(u)=k(u)+c a(u)
$$

where $c=\frac{\bar{S}(u)}{\tau_{S}}$.

\section{ENTROPY MINIMIZATION}

The formulation in Equation 8 is important since we can now view the recovery of $k(u)$ in an entropy minimisation setting. Here we employ an entropy minimization. Along these lines, our strategy is to estimate $c$. Once $c$ is at hand, $a(u)$ and $b(u)$ can be estimated using linear regression. With $c, a(u)$ and $b(u)$ at hand the specular coefficient can be obtained in a straightforward manner from Equation 8.

For a homogeneous reflectance region in the image, $\hat{S}(\cdot, u)$ is constant, with $k(u)$ and $g(u)$ varying accordingly. This implies that every pixel in the homogenous region should correspond to a line in the $a(u)-b(u)$ space. This is illustrated in Figure 1. Note that all these share the slope $c$. By projecting the points on the direction of any line in the $a(u)-b(u)$, we can appreciate that the frequency distribution of these has a skew which is minimum when the intersecting line is perpendicular to $b(u)=k(u)+c a(u)$.

This is illustrated in Figure 1. Note that, this implies that the entropy for the frequency distribution for the pixel projections of the set of intersecting lines is minimum for the line whose slope is $\frac{1}{c}$. This suggests the use of the Shannon entropy for the recovery of $c$.

Thus, we aim at recovering the angle $\theta$ on which the projected points have a minimal entropy. Hence, we project every point corresponding to the pixels in the homogeneous reflectance image region onto the line with slope given by $\tan \theta$. The distance is given by

$$
d(u, \theta)=a(u) \cos (\theta)+b(u) \sin (\theta)
$$

Here, we use Shannon's entropy to quantify the degree of skewness for the distribution of projected points. This can be done computationally by making use of a histogram. To do this, we equally divide the interval $[\min d(u, \theta), \max d(u, \theta)]$ of $d(u, \theta)$ into $K$ bins such that the $i^{t h}$ bin comprises those pixels in the range $[(i-1) r, i r)$, where $r=\max d(u, \theta)-\min d(u, \theta)$. We then take the cumulative and view it as the probability of a point in the bin indexed $i$ falling in the interval under consideration. This is $p(i \mid \theta)=\frac{N_{i}(\theta)}{N_{\text {total }}}$, where $N_{\text {total }}$ is the number of pixels in the homogenous reflectance region under study and $N_{i}(\theta)$ corresponds to the number of pixels in the interval $[(i-1) r, i r)$. With these ingredients, the Shannon entropy is given by

$$
\eta(\theta)=-\sum_{i} p(i \mid \theta) \log (p(i \mid \theta))
$$

To implement the algorithm, we proceed as follows. Firstly, we note that, for imaging spectroscopy data, we can get the illuminant power spectrum $L(\lambda)$ making use of the method in [17]. Once the illuminant power spectrum is in hand, we apply $k$-means to the vectors given by $R\left(\lambda_{i}, u\right)$. This yields a set of clusters whose pixels are then further selected through the application of a dichromatic plane [18].

This is as the spectra of all pixels of a uniform reflectance can be shown to span a two-dimensional subspace. For more details, see [19]. Thus, we select a subset of each cluster such that the corresponding pixels are those that deviate less from the dichromatic plane. This is done by performing a singular value decomposition as proposed in [18].

We use these pixels as the input to our method. To recover the variable $c$ in Equation 8, we commence by applying a linear regression on Equation 6. With $a(u)$, $b(u)$ in hand, we perform a search over the values of the entropy in Equation 10 by varying the angle $\theta$ and selecting the value which yields the lowest $\eta(\theta)$. Once the $c$ has been selected, we recover the value of $k(u)$ for every pixel in each of the $k$ clusters.

\section{EXPERIMENTS}

In this section, we illustrate the utility of our method for specularity removal. To this end, we compare our results to those yielded by the method in [20]. The alternative is a specularity removal method based upon chromaticity. For our experiments, we have modified the method slightly so as to work with $N$ bands instead of three so as to account for the fact that here we work 

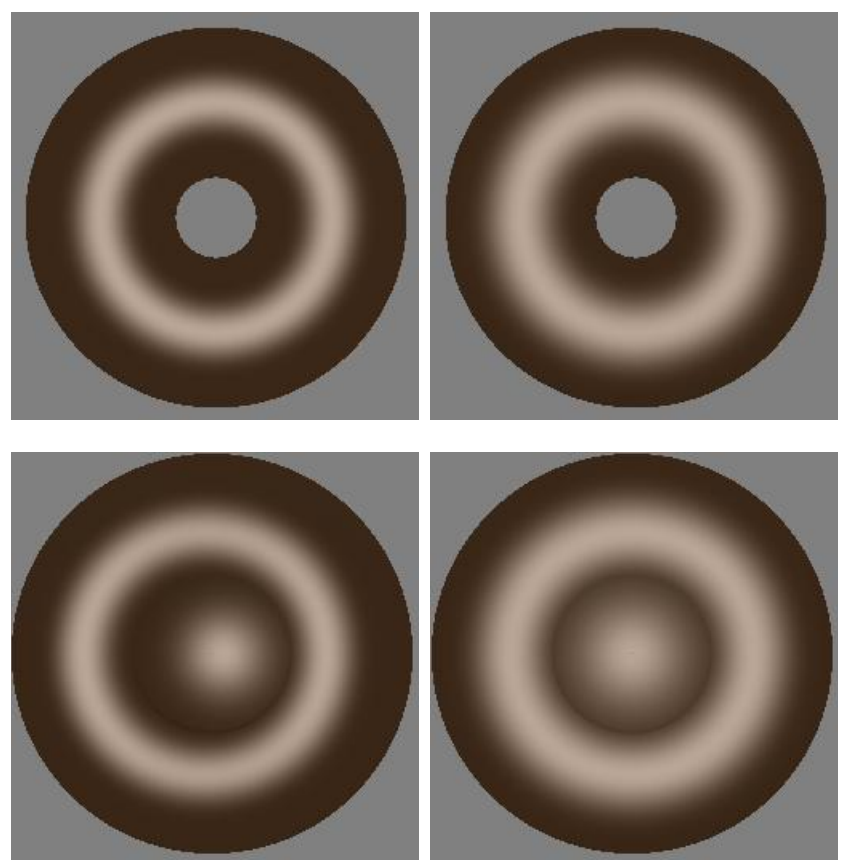

Fig. 2. Trichromatic pseudocolour images for the torus and the dome. Left-hand column: Imagery rendered using the Cook and Torrance model; Right-hand column: Images rendered using Phong's model.

with imaging spectroscopy data rather than trichromatic imagery.

In all our experiments, we have set the number of bins $K$ to 64 and the number of clusters $k$ to 20 . Here, we use two datasets. The first of these is comprised by synthetically generated imagery. The second of these consists of real-world images. For the synthetic data, we use computer-generated imagery. To produce the images, we have used two reflectance models widely used in the literature. These are the Phong model [21] and the CookTorrance model [22]. Our choice hinges in the nature of these, the former is a phenomenological one which is widely employed in graphics, whereas the latter is a physics-based model which captures specularity through a Beckmann distribution [23].

To render the imagery, we use 3D models of two simple shapes, i.e. a torus and parabolic dome. For both models, the shading factor is given by

$$
g(u)=\langle\vec{N}, \vec{L}\rangle
$$

where $\vec{N}$ is the surface normal at pixel $u, \vec{L}$ is the light source direction and $\langle\cdot, \cdot\rangle$ denotes the dot product.

The image radiance is then given by the dichromatic model with the specular term given by either of the two
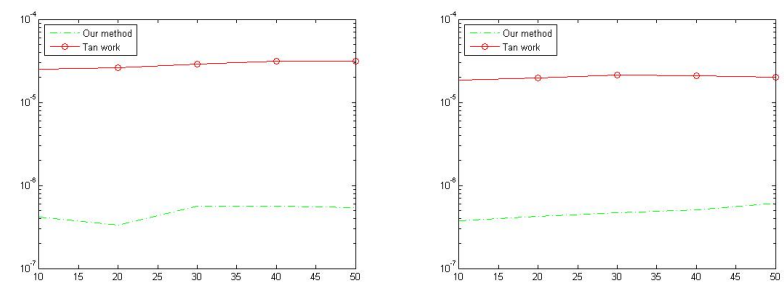

Fig. 3. The error as a function of illumination direction for the model of Cook and Torrance. The green line corresponds to our method, whereas the red line shows the result yielded by the method in [20]. The left hand panel shows the error plots for the torus. The right-hand panel shows the plots for the dome.

models under consideration. Thus, we have

$$
I(\lambda, u)=\sigma_{s} g(u) S(\lambda, u) L(\lambda)+\sigma_{d} k(u) L(\lambda)
$$

where $\sigma_{s} g(u)+\sigma_{d} k(u)=1$ Here $\sigma_{s}$ and $\sigma_{d}$ are the parameters that control the intensity of the diffuse specular components. In Equation 11, $k(u)$ is given by the reflectance model of choice. For the Phong model, we have

$$
k(u)=0.5 \cos (\theta)+0.5 \cos (\theta)^{\eta}
$$

where $\theta=\arccos (\vec{N} \cdot \vec{L})$. For our experiments, we have generated synthetic images with values of shininess in the interval $\eta \in[0.5,5]$.

For the Cook and Torrance model we have

$$
k(u)=0.5(\vec{N} \cdot \vec{H})+0.5 \frac{\mathcal{D G \mathcal { F }}}{\pi(\vec{N} \cdot \vec{V})(\vec{N} \cdot \vec{L})}
$$

where $\vec{H}$ is the half-way vector and the terms $\mathcal{D}, \mathcal{G}$ and $\mathcal{F}$ are the microfacet-slope distribution, geometric factor and Fresnel term, respectively, and are given by

$$
\begin{aligned}
\mathcal{D} & =\frac{\exp \left\{-\tan \left(\frac{\arccos (\vec{N} \cdot \vec{H})}{m_{p}}\right)^{2}\right\}}{4 m_{p}^{2}(\vec{N} \cdot \vec{H})^{4}} \\
\mathcal{G} & =\min \left\{1, \frac{2(\vec{N} \cdot \vec{H})(\vec{N} \cdot \vec{V})}{(\vec{V} \cdot \vec{H})}, \frac{2(\vec{N} \cdot \vec{H})(\vec{N} \cdot \vec{L})}{(\vec{V} \cdot \vec{H})}\right\} \\
\mathcal{F} & =0.5 \frac{\sin \left((\vec{L} \cdot \vec{H})-\arcsin \left(\frac{\sin (\vec{L} \cdot \vec{H})}{m_{p}}\right)\right)^{2}}{\sin \left((\vec{L} \cdot \vec{H})+\arcsin \left(\frac{\sin (\vec{L} \cdot \vec{H})}{m_{p}}\right)\right)^{2}}
\end{aligned}
$$

where we have denoted the microfacet-slope by $m_{p}$. Here, we have generated images by varying the microfacet-slope parameter $m_{p}$ from 0.20 to 0.40 in increments of 0.05 and varied the illuminant direction $\vec{L}$. 
Here, the reflectance $S(\lambda, u)$ of the material has been collected in-house using an spectrometer. For our imagery, we have used the reflectance for a number of materials, such as wood, skins, etc. In Figure 2, we show two sample images rendered using one of our wood samples. In the figure, we show the colour images yielded by the application of the colour matching functions in [24] according to the device-independent CIE standard [25]. In the panels, for the Cook and Torrrance model, we have set $m_{p}$ to 0.3 . For Phong's model, we use a shininess factor $\eta$ of 5 and set $\vec{L}^{\prime}=\vec{V}$.

To provide a quantitative analysis, we use the specular coefficient $k(u)$ from the ground truth and employ the absolute error between this and the specularities recovered by our method and the alternative for each of the input images. Once the mean absolute error per image is in hand, we compute the mean of these quantities for the whole of our datasets for both, Phong's and Torrance-Sparrow models. In Figures 3 and 4 we show the error as a function of illuminant direction $\vec{L}$ such that $\arccos \langle\vec{V}, \vec{L}\rangle \in\left[0,50^{\circ}\right]$. Since the error magnitudes vary dramatically, we have used a logarithmic scale.

Note that, from the figures, we can appreciate that our method yields, in some cases, an improvement of more than an order of magnitude with respect to the alternative. This is further confirmed in Figures 5 and 6 where we plot the error as a function of $\sigma_{s}$. Again, we have used a logarithmic scale. In the figures, the trend is similar, with the difference between the two methods decreasing as the specular component increases.

We now turn our attention to the error with respect to the shininess $\eta$ and the microfacet slpe $m_{p}$. These parameters are important since they determine the specular component induced by both, Phong's and the Cook and Torrance model. In Figure 8, we plot the error as a
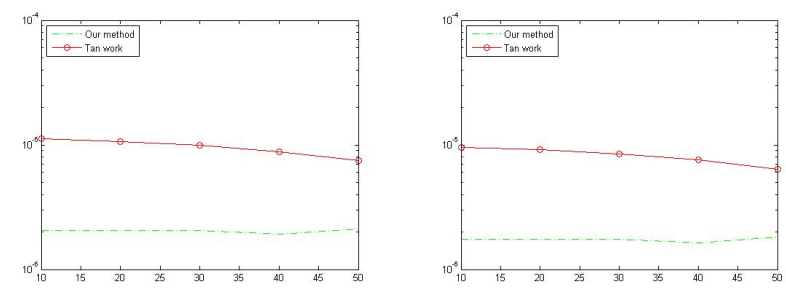

Fig. 4. The error as a function of illumination direction for Phong's model. The green line corresponds to our method, whereas the red line shows the result yielded by the method in [20]. The left hand panel shows the error plots for the torus. The right-hand panel shows the plots for the dome.
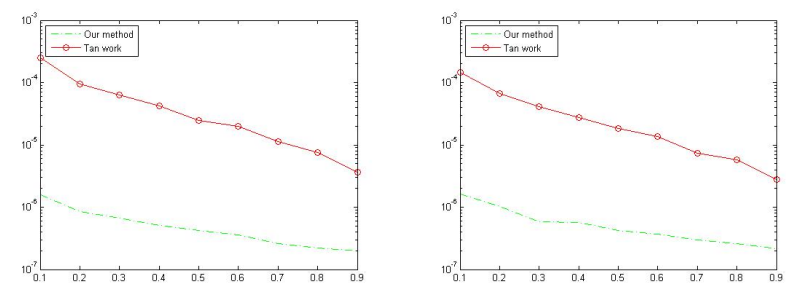

Fig. 5. The error as a function of $\sigma_{s}$ for the Torrance and Sparrow model. The green line corresponds to our method, whereas the red line shows the result yielded by the method in [20]. The left hand panel shows the error plots for the torus. The right-hand panel shows the plots for the dome.

function of shininess. In Figure 7 we repeat the sequence for the microfacet slope.

From the figures, we can conclude that our method is effective over a wide range of parameters and viewing directions for both models. Maybe the most noticeable aspect of the last set of figures is that, for our method, as the shininess increases, the error decreases. In contrast, the alternative has an error that is almost constant with respect to the shininess parameter.

Finally, we turn our attention to the real world imagery. To illustrate the utility of our method, we use two hyperspectral images. Our two images correspond to a portrait and a wooden toy, both composed of spectra sampled at intervals of $10 \mathrm{~nm}$ in the range between 430 $\mathrm{nm}$ and $650 \mathrm{~nm}$, i. e. 23 bands. To process these images, and as mentioned earlier, we commence by applying the method in [26] so as to recover the illuminant power spectrum. In Figures 9 and 10 we show, in the left-hand column the portrait and the detail of our toy image reproduced using the pseudocolour yielded by the colour matching functions according to the deviceindependent CIE standard [25]. We have used a detail
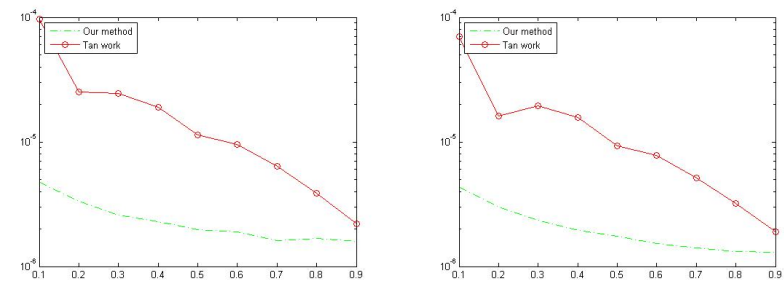

Fig. 6. The error as a function of $\sigma_{s}$ for Phong's model. The green line corresponds to our method, whereas the red line shows the result yielded by the method in [20]. The left hand panel shows the error plots for the torus. The right-hand panel shows the plots for the dome. 

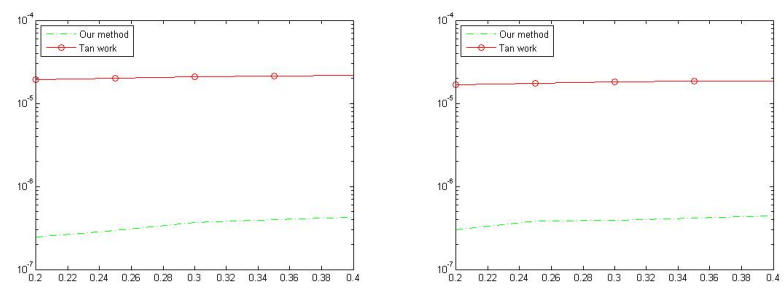

Fig. 7. The error as a function of microfacet slope for the Cook and Torrance model. The green line corresponds to our method, whereas the red line shows the result yielded by the method in [20]. The left hand panel shows the error plots for the torus. The right-hand panel shows the plots for the dome.

of the toy so as to provide a better illustration of the capacity of the algorithm for specularity removal. In the second column, we show the specularities yielded by our method. The third column corresponds to those recovered by the alternative. The fourth and fifth columns show the pseudocolour for the diffuse radiance recovered by both, our method and that in [20], respectively.

From the imagery, we can conclude that our method delivers imagery in better accordance with the expected appearance of both, the specularities and the diffuse radiance. This is particularly evident for the toy detail, where the pseudocolour for the diffuse radiance appears noisy. This also shows an interesting consequence of specularity removal in imaging spectroscopy. Nonetheless the specular coefficient in Equation 2 is an additive one, the illuminant power spectrum is a multiplicative factor affecting both, diffuse and specular reflection. Thus, errors in the specularity removal process may duly affect the diffuse radiance when the power of the light plays an important role in the image formation process by, effectively, magnifying the error.
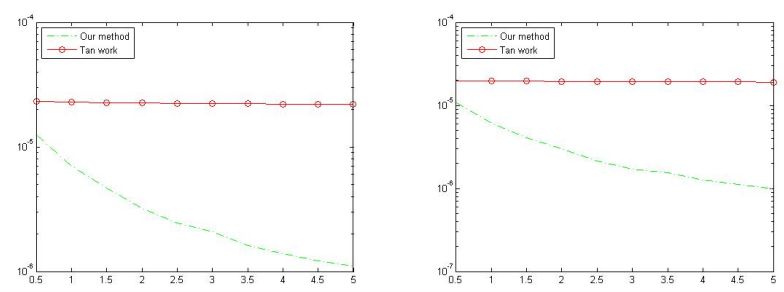

Fig. 8. The error as a function of shininess for Phong's model. The green line corresponds to our method, whereas the red line shows the result yielded by the method in [20]. The left hand panel shows the error plots for the torus. The right-hand panel shows the plots for the dome.

\section{Conclusions}

In this paper, we have proposed a method to effect specularity removal from imaging spectroscopy data. We have done this by making use of the dichromatic model so as to cast the problem in a linear regression setting. We have shown how the average radiance at each pixel can be used to map the spectra onto a two-dimensional space. This mapping allows for the use of a Shannon's entropy as a metric that can be employed to recover the slope of a line described by a linear regressor. We have provided a quantitative analysis making use of synthetic data and illustrated the effectiveness of our approach for specularity removal on real-world imagery. We have also provide comparison with an alternative.

\section{REFERENCES}

[1] H. M. G. Stokman and T. Gevers, "Detection and classification of hyper-spectral edges," in British Machine Vision Conference, 1999.

[2] E. Angelopoulou, "Objective colour from multispectral imaging," in European Conf. on Computer Vision, 2000, pp. 359374.

[3] J. Y. Chang, K. M. Lee, and S. U. Lee, "Shape from shading using graph cuts," in Proc. of the Int. Conf. on Image Processing, 2003.

[4] Z. Fu, T. Caelli, N. Liu, and A. Robles-Kelly, "Boosted band ratio feature selection for hyperspectral image classification," in Proc. Intl. Conf. Pattern Recognition, vol. 1, 2006, pp. 10591062.

[5] Z. Fu, R. Tan, and T. Caelli, "Specular free spectral imaging using orthogonal subspace projection," in Proc. Intl. Conf. Pattern Recognition, vol. 1, 2006, pp. 812-815.

[6] G. Brelstaff and A. Blake, "Detecting specular reflection using lambertian constraints," in Int. Conference on Computer Vision, 1988, pp. 297-302.

[7] S. Nayar and R. Bolle, "Reflectance based object recognition," International Journal of Computer Vision, vol. 17, no. 3, pp. 219-240, 1996.

[8] S. Lin and H. Shum, "Separation of diffuse and specular reflection in color images," in IEEE Conf. on Computer Vision and Pattern Recognition, 2001.

[9] G. Klinker, S. Shafer, and T. Kanade, "A physical approach to color image understanding," Intl. Journal of Computer Vision, vol. 4, no. 1, pp. 7-38, 1990.

[10] H. Ragheb and E. R. Hancock, "A probabilistic framework for specular shape-from-shading," Pattern Recognition, vol. 36, no. 2, pp. 407-427, 2003.

[11] C. Novak and S. Shafer, "Anatomy of a color histogram," in Proc. of the IEEE Conference on Computer Vision and Pattern Recognition, 1992, pp. 599-605.

[12] Y. Sato and K. Ikeuchi, "Temporal-color space analysis of reflection," in Proc. of the IEEE Conference on Computer Vision and Pattern Recognition, 1993, p. 70576.

[13] S. A. Shafer, "Using color to separate reflection components," Color Research and Applications, vol. 10, no. 4, pp. 210-218, 1985. 

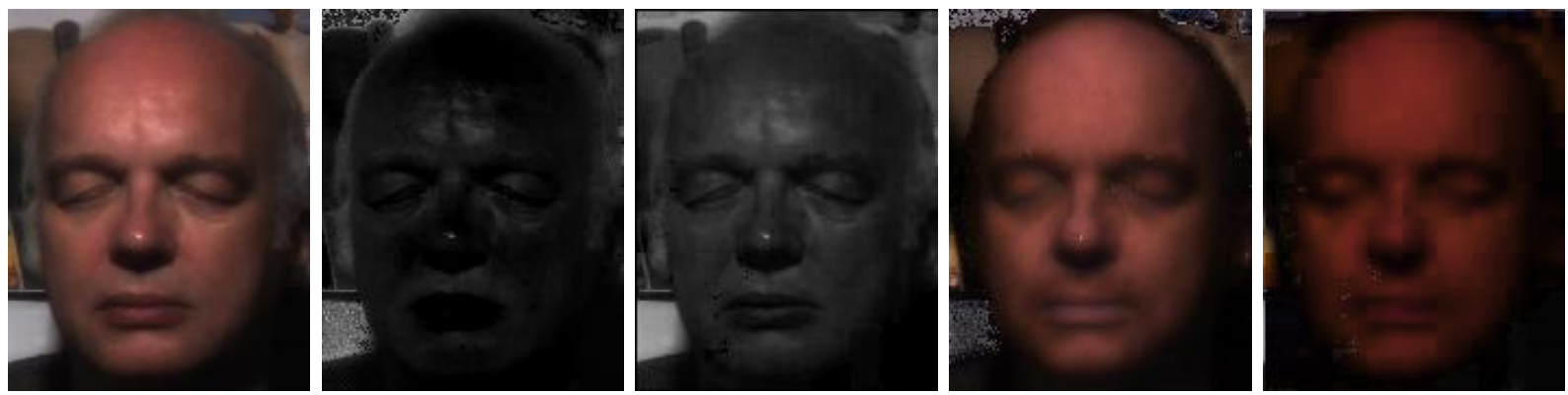

Fig. 9. From left-to-right: Input pseudocolour image of the portrait used in our experiments, specularities recovered by our method, specularities yielded by the alternative, the pseudocolour imagery for the diffuse radiance recovered using our method, the pseudocolour imagery for the diffuse radiance yielded by the alternative.
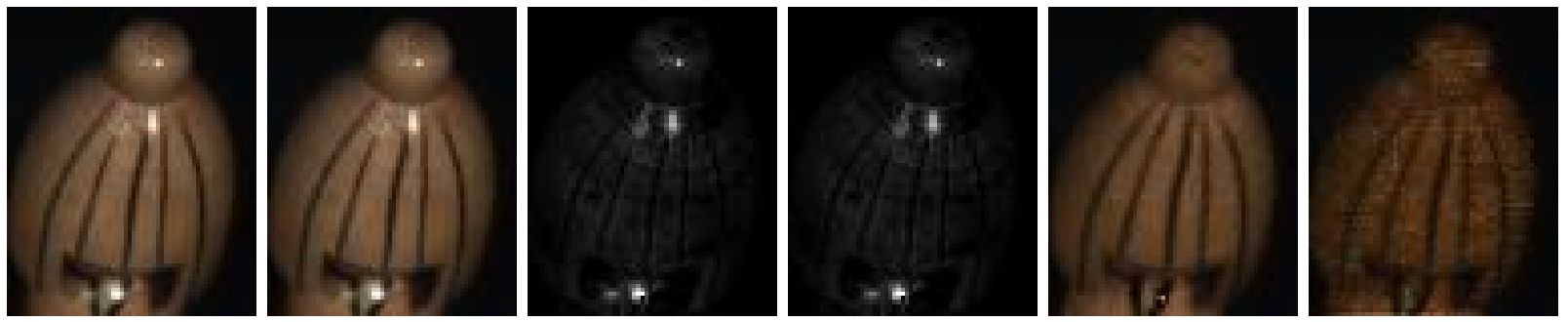

Fig. 10. From left-to-right: Pseudocolour image of the toy used in our experiments, pseudocolour for the detail used as input to our method and the alternative, specularities recovered by our method, specularities yielded by the alternative, the pseudocolour imagery for the diffuse radiance recovered using our method, the pseudocolour imagery for the diffuse radiance yielded by the alternative.

[14] S. Umeyama and G. Godin, "Separation of diffuse and specular components of surface reflection by use of polarization and statistical analysis of images," IEEE Trans. Pattern Analysis and Machine Intelligence, vol. 26, no. 5, pp. 639-647, 2004.

[15] R. T. Tan and K. Ikeuchi, "Separating reflection components of textured surfaces using a single image," IEEE Transactions on Pattern Analysis and Machine Intelligence, vol. 27, no. 2, pp. 178-193, 2005.

[16] G. D. Finlayson, M. S. Drew, and C. Lu, "Intrinsic images by entropy minimization," in European Conf. on Computer Vision, 2004, pp. 582-595.

[17] C. P. Huynh and A. Robles-Kelly, "A solution of the dichromatic model for multispectral photometric invariance," International Journal of Computer Vision, vol. 90, no. 1, pp. 1-27, 2010.

[18] S. Tominanga and B. A. Wandell, "Standard surface-reflectance model and illuminant estimation," Journal of the Optical Society of America A, no. 6, pp. 576-584, 1989.

[19] G. D. Finlayson and G. Schaefer, "Solving for colour constancy using a constrained dichromatic reflection model," International Journal of Computer Vision, vol. 42, no. 3, pp. 127-144, 2001.

[20] R. T. Tan, K. Nishino, and K. Ikeuchi, "Separating reflection components based on chromaticity and noise analysis," IEEE Trans. Pattern Analysis and Machine Intelligence, vol. 26, no. 10, pp. 1373-1379, 2004.

[21] B. T. Phong, "Illumination for computer generated pictures," Communications of the ACM, vol. 18, no. 6, pp. 311-317, 1975.

[22] R. L. Cook and K. E. Torrance, "A reflectance model for computer graphics," ACM Trans. on Graphics, vol. 1, no. 1, pp. 7-24, 1982.

[23] P. Beckmann and A. Spizzochino, The Scattering of Electro- magnetic Waves from Rough Surfaces. Pergamon, New York, 1963.

[24] W. S. Stiles and J. M. Burch, "N.P.L. colour-matching investigation: Final report 1958," Optica Acta, vol. 6, pp. 1-26, 1959.

[25] CIE, Commission Internationale de lEclairage Proceedings, 1931. Cambridge University Press, 1932.

[26] C. P. Huynh and A. Robles-Kelly, "Hyperspectral imaging for skin recognition and biometrics," in International Conference on Image Processing, 2010. 